Letter to the Editor

\title{
Dexamethasone implant for the treatment of persistent diabetic macular oedema despite long-term treatment with bevacizumab
}

\author{
Aaron W Joe PhD FRCSC, ${ }^{1}$ Sanjeewa S Wickremasinghe DMedSc FRANZCO, ${ }^{2}$ Mark C \\ Gillies PhD FRANZCO, ${ }^{2}$ Vuong Nguyen PhD,${ }^{1}$ Lyndell L Lim FRANZCO, ${ }^{2}$ Hemal Mehta MA \\ FRCOphth ${ }^{1,3}$ and Samantha Fraser-Bell PhD FRANZCO ${ }^{1}$
}

1. The Save Sight Institute, Sydney Medical School, University of Sydney, Sydney, Australia

2. Centre for Eye Research Australia, Royal Victorian Eye and Ear Hospital, University of Melbourne, Melbourne, Australia

3. Royal Free London NHS Foundation Trust, United Kingdom

Correspondence: Aaron W. Joe, Macula Research Group, Save Sight Institute, University of Sydney, 8 Macquarie Street, Sydney, NSW 2000, Australia

Email: dr.aaronjoe@gmail.com

Received 16 April 2018; accepted 2 August 2018

Conflict of interest: None

Funding sources: AJ: none, SW: Financial Support: Australian National Health and Medical Research Council (NHMRC) and Novartis; Recipient of Gifts, Honoraria, Travel Reimbursement or Patent: Allergan, Bayer and Novartis. MC: Consultant and Financial Support: Allergan, Bayer and Novartis. VN: None. LL: Consultant and Financial Support:

This is the author manuscript accepted for publication and has undergone full peer review but has not been through the copyediting, typesetting, pagination and proofreading process, which may lead to differences between this version and the Version of Record. Please cite this article as doi: $10.1111 /$ ceo.13371

This article is protected by copyright. All rights reserved. 
Abbvie, Allergan and Bayer; Financial Support: Novartis. HM: Consultant and Financial Support: Allergan, Bayer and Novartis. SF-B: Consultant and Financial Support: Allergan, Bayer and Novartis

We conducted a prospective study to assess the benefit of switching from bevacizumab to dexamethasone $0.7 \mathrm{mg}$ intravitreal implant (DEX-implant) (Ozurdex, Allergan) in eyes with persistent diabetic macular oedema (DMO).

Although anti-VEGF therapy is considered first line treatment for centre-involving DMO, DMO does not fully resolve in a significant proportion of eyes receiving regularlydosed, anti-VEGF treatment. ${ }^{1,2}$ Our study examined switching eyes from bevacizumab monotherapy to DEX-implant monotherapy rather than adding the DEX-implant to antiVEGF therapy as in DRCRnet protocol U.

Patients were enrolled at 2 Australian public hospital clinics (December 2013 to February 2017). Inclusion criteria included eyes with central macular thickness (CMT) $>300 \mu \mathrm{m} 4$ weeks after at least 6 consecutive months of treatment with bevacizumab and BCVA 20-78 LogMAR letters (Snellen equivalent $=6 / 120$ to 6/9). Both phakic and pseudophakic eyes were included. Exclusion criteria included uncontrolled glaucoma or glaucoma controlled with more than 1 medication, vision loss due to other causes and macular oedema due to other causes.

At time of enrollment, treatment with bevacizumab was discontinued. An 8week washout period then ensued, immediately followed by the baseline visit. The washout period was necessary to rule out synergistic effects between bevacizumab and intraocular steroid. ${ }^{3}$ DEX-implant was administered at the baseline visit, and patients were examined every 4 weeks. Retreatment with DEX-implant was considered after 16 weeks if CMT was $>300 \mu \mathrm{m}$ and/or BCVA $<6 / 7.5$. The primary outcome was the 
proportion of eyes with $\mathrm{CMT}<300 \mu \mathrm{m}$ at 6 months. Secondary outcomes included mean change in BCVA and adverse events.

A total of 16 eyes from 16 patients were enrolled, 15 completed the study. Twelve of 16 subjects were male and the mean age was 65.3 years. Two subjects had type 1 diabetes, 12 had type 2, and 2 were not specified. The average duration of diabetes was 18.1 years, and 12 subjects required insulin. The majority (11 of 16 eyes) had undergone previous laser pan-retinal photocoagulation for treatment of proliferative diabetic retinopathy. The vast majority demonstrated diffuse leak on FFA at baseline. Study eyes had received a mean of 14.3 bevacizumab injections (range $6-36$ ). Seven eyes had previously received 2 years of intensive bevacizumab therapy as participants in the BEVORDEX study (where eyes were treated with bevacizumab every 4 weeks, $\mathrm{PRN})^{4}$. At the time of enrollment (prior to the washout period) mean CMT was $541 \mu \mathrm{m}$ (95\% CI: $467-641 \mu \mathrm{m}$ ), increasing to $569 \mu \mathrm{m}$ at baseline (after washout, $\mathrm{p}=0.17$ ). Four weeks after administration of the first DEX implant, mean CMT significantly improved to $343 \mu \mathrm{m}$ ( $95 \%$ CI $270-416 \mu \mathrm{m}, \mathrm{p} \leq 0.002$, Figure 1, Table 1) but increased again at week 16 to $433 \mu \mathrm{m}$ ( $95 \%$ CI $358-508 \mu \mathrm{m}$ ) (Figure 1, Table 1). After week 16, 63\% (10 of 16 eyes) received a second DEX-implant. At 24 weeks, $47 \%$ of eyes ( 7 of 15) achieved our primary outcome of CMT $<300 \mu \mathrm{m}$. The average central macular thickness was $349 \mu \mathrm{m}$ (95\% CI $275-422 \mu \mathrm{m})$, significantly less than baseline $(p=0.0003)$. Mean reduction in CMT was $220 \mu \mathrm{m}$ (95\% CI $118-32 \mu \mathrm{m})$ (Figure 1).

The average BCVA change was +3.9 logMAR letters (95\% CI -1.0 - 8.9 logMAR letters) at the study endpoint ( $p=0.10) . A \geq 5$ letter improvement in BCVA occurred in $56 \%$ ( 9 eyes) and 3 eyes achieved BCVA $\geq 10$ logMAR letters. Elevation in IOP $>10 \mathrm{mmHg}$ occurred in 3 eyes (19\%): 1 required incisional glaucoma surgery and 2 eyes had IOP controlled with latanoprost. The eye which required glaucoma surgery lost 13 logMAR letters even though CMT was reduced by $347 \mu \mathrm{m}$. Of the 12 phakic eyes at 
baseline, cataract progression was observed in 1 eye during the study, and in 2 eyes at a safety visit 9 months after baseline.

Similar to DRCRnet Protocol U, we identified little improvement in mean BCVA despite significant anatomical improvement. ${ }^{5}$ Chronicity of DMO may play a role in limiting visual gains. ${ }^{2}$ We noticed an increase in CMT at week 16 , suggesting that earlier retreatment may be warranted to prevent rebound DMO. However, it is as yet unknown whether a less oedematous macula yields better long term visual outcomes than a persistently thickened one.

A mean of 14.3 (range 6-36) anti-VEGF were delivered prior to switching in our study. Despite the long period of anti-VEGF treatment, there was still significant reduction in CMT after switching to the DEX-implant, although this did not always translate into visual benefit. Limitations include the relatively small sample size and absence of an anti-VEGF monotherapy arm for comparison. Results may also be different with other approved anti-VEGF agents.

We found that DEX-implant can reduce DMO that has not responded to between 6 months and 2 years of regular bevacizumab monotherapy. Although over half of our patients gained 5 letters or more, similar to DRCRnet Protocol $U$, some eyes lost vision, resulting in no significant overall gain in mean BCVA. There is an urgent need to identify whether reducing DMO without short-term gain in vision is beneficial in the long-term. 


\section{FIGURES:}

Figure 1.

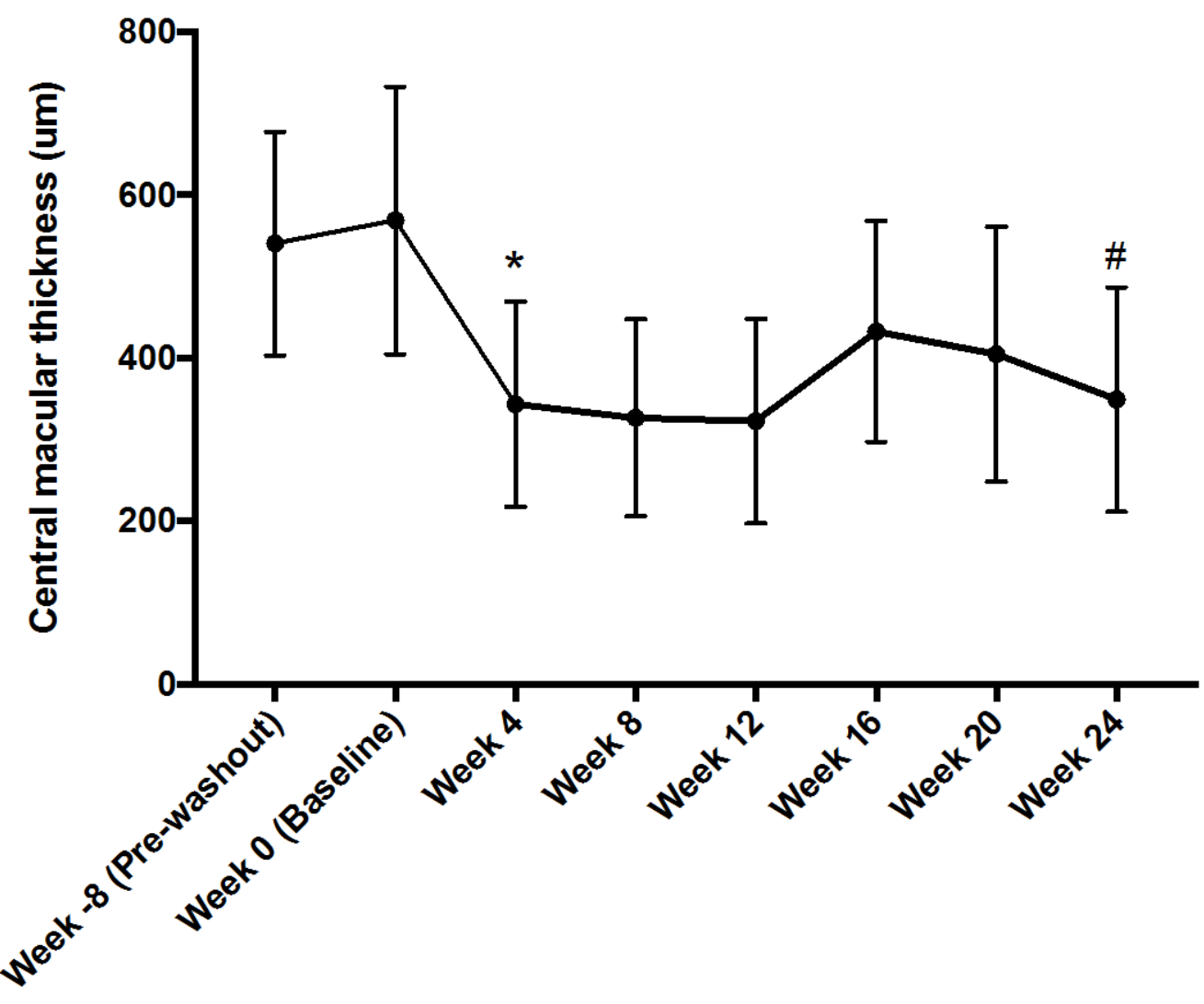

Figure 1: Mean central macular thickness $(\mu \mathrm{m})$ during the study. Pre-washout values were the last CMT measurements while on bevacizumab. Baseline measurements were performed after a 2-month washout. Dexamethasone $0.7 \mathrm{mg}$ implant was administered to all eyes at baseline. Re-treatment was given on a PRN basis at week 16 or after. Error bars represent standard deviation. $* p=0.002$ compared with baseline. $\# p=$ 0.0007 compared with baseline. 
Table 1: Values for mean central macular thickness $(\mu \mathrm{m})$ and mean BCVA (LogMAR letters) during the study.

Dexamethasone $0.7 \mathrm{mg}$ implant was administered at baseline. Re-treatment was given on a PRN basis after week 16. CMT (Central macular thickness), SD (Standard deviation), CI (Confidence interval), BCVA (Best corrected visual acuity).

\begin{tabular}{|c|c|c|c|c|c|c|c|}
\hline & Baseline & 4 weeks & 8 weeks & 12 weeks & 16 weeks & 20 weeks & 24 weeks \\
\hline Mean CMT ( $(\mu \mathrm{m})$ & 569 & 343 & 327 & 323 & 433 & 405 & 349 \\
\hline$S D$ & 164 & 126 & 121 & 126 & 135 & 156 & 138 \\
\hline Upper $95 \%$ CI of mean & 656 & 416 & 391 & 390 & 508 & 488 & 422 \\
\hline Lower $95 \%$ CI of mean & 482 & 270 & 262 & 256 & 358 & 322 & 275 \\
\hline Mean BCVA (LogMAR letters) & 58 & 65 & 62 & 62 & 57 & 61 & 62 \\
\hline$S D$ & 15 & 12 & 14 & 14 & 13 & 15 & 17 \\
\hline Upper $95 \%$ CI of mean & 66 & 71 & 69 & 69 & 65 & 69 & 71 \\
\hline Lower $95 \%$ CI of mean & 50 & 58 & 54 & 55 & 50 & 52 & 53 \\
\hline
\end{tabular}




\section{Acknowledgements}

The authors would like to acknowledge Dr Jeremy Smith, Mr. Paul Power and Ms.

Anette Kifly as safety monitors for the trial, and Ms. Jaclyn Brant, Ms. Roxy Medina and Ms. Sutha Sanmugasundaram for their assistance with data collection and maintaining the databases.

\section{REFERENCES}

1. Bressler NM, Beaulieu WT, Glassman AR, et al. Persistent Macular Thickening Following Intravitreous Aflibercept, Bevacizumab, or Ranibizumab for Central-Involved Diabetic Macular Edema With Vision Impairment: A Secondary Analysis of a Randomized Clinical Trial. JAMA Ophthalmol 2018;136(3):257-69.

2. Brown DM, Nguyen QD, Marcus DM, et al. Long-term outcomes of ranibizumab therapy for diabetic macular edema: the 36-month results from two phase III trials: RISE and RIDE. Ophthalmology 2013;120(10):2013-22.

3. Mehta $\mathrm{H}$, Hennings $\mathrm{C}$, Gillies $\mathrm{MC}$, et al. Anti-vascular endothelial growth factor combined with intravitreal steroids for diabetic macular oedema. Cochrane Database Syst Rev 2018;4:Cd011599.

4. Gillies MC, Lim LL, Campain A, et al. A randomized clinical trial of intravitreal bevacizumab versus intravitreal dexamethasone for diabetic macular edema: the BEVORDEX study. Ophthalmology 2014;121(12):2473-81.

5. Maturi RK, Glassman AR, Liu D, et al. Effect of Adding Dexamethasone to Continued Ranibizumab Treatment in Patients With Persistent Diabetic Macular Edema: A DRCR Network Phase 2 Randomized Clinical Trial. JAMA Ophthalmol 2018;136(1):2938. 


\section{University Library}

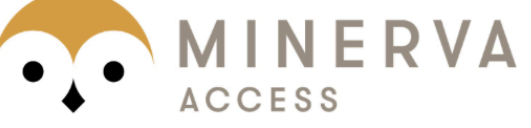

A gateway to Melbourne's research publications

Minerva Access is the Institutional Repository of The University of Melbourne

Author/s:

Joe, AW;Wickremasinghe, SS;Gillies, MC;Vuong, N;Lim, LL;Mehta, H;Fraser-Bell, S

Title:

Dexamethasone implant for the treatment of persistent diabetic macular oedema despite long-term treatment with bevacizumab

Date:

2019-03-01

\section{Citation:}

Joe, A. W., Wickremasinghe, S. S., Gillies, M. C., Vuong, N., Lim, L. L., Mehta, H. \& FraserBell, S. (2019). Dexamethasone implant for the treatment of persistent diabetic macular oedema despite long-term treatment with bevacizumab. CLINICAL AND EXPERIMENTAL OPHTHALMOLOGY, 47 (2), pp.287-289. https://doi.org/10.1111/ceo.13371.

Persistent Link:

http://hdl.handle.net/11343/284351 